\title{
Resolving the distance controversy for Sharpless 269
}

\section{A possible kink in the outer arm}

\author{
L. H. Quiroga-Nuñez ${ }^{1,2}$, K. Immer ${ }^{2}$, H. J. van Langevelde ${ }^{2,1}$, M. J. Reid ${ }^{3}$, and R. A. Burns ${ }^{2}$ \\ ${ }^{1}$ Leiden Observatory, Leiden University, PO Box 9513, 2300 RA Leiden, The Netherlands \\ e-mail: quiroganunez@strw. leidenuniv.nl \\ 2 Joint Institute for VLBI ERIC (JIVE), Oude Hoogeveensedijk 4, 7991 PD Dwingeloo, The Netherlands \\ 3 Harvard-Smithsonian Center for Astrophysics, 60 Garden Street, Cambridge, MA 02138, USA
}

Received 27 November 2018 / Accepted 24 March 2019

\begin{abstract}
Context. Sharpless 269 (S 269) is one of a few HII regions in the outer spiral arm of the Milky Way with strong water maser emission. Based on data from the Very Long Baseline Interferometry (VLBI) Exploration of Radio Astrometry (VERA) array, two parallax measurements have been published, which differ by nearly $2 \sigma$. Each distance estimate supports a different structure for the outer arm. Moreover, given its large Galactocentric radii, S 269 has special relevance as its proper motion and parallax have been used to constrain the Galactic rotation curve at large radii.

Aims. Using recent Very Long Baseline Array (VLBA) observations, we accurately measure the parallax and proper motion of the water masers in S 269. We interpret the position and motion of S 269 in the context of Galactic structure, and possible optical counterparts.

Methods. S 269's $22 \mathrm{GHz}$ water masers and two close by quasars were observed at 16 epochs between 2015 and 2016 using the VLBA. We calibrated the data by inverse phase referencing using the strongest maser spot. The parallax and proper motion were fitted using the standard protocols of the Bar and Spiral Structure Legacy survey.

Results. We measure an annual parallax for S 269 of $0.241 \pm 0.012$ mas corresponding to a distance from the Sun of $4.15_{-0.20}^{+0.22} \mathrm{kpc}$ by fitting four maser spots. The mean proper motion for $\mathrm{S} 269$ was estimated as $0.16 \pm 0.26 \mathrm{mas} \mathrm{yr}^{-1}$ and $-0.51 \pm 0.26 \mathrm{mas}^{-1}$ for $\mu_{\alpha} \cos \delta$ and $\mu_{\delta}$ respectively, which corresponds to the motion expected for a flat Galactic rotation curve at large radius. This distance estimate, Galactic kinematic simulations and observations of other massive young stars in the outer region support the existence of a kink in the outer arm at $l \approx 140^{\circ}$. Additionally, we find more than 2000 optical sources in the Gaia DR2 catalog within $125 \mathrm{pc}$ radius around the 3D position of the water maser emission; from those only three sources are likely members of the same stellar association that contains the young massive star responsible for the maser emission (S 269 IRS 2w).
\end{abstract}

Key words. masers - astrometry - stars: massive - stars: early-type - open clusters and associations: individual: S 269 Galaxy: structure

\section{Introduction}

The Very Long Baseline Interferometry (VLBI) Exploration of Radio Astrometry $\left(\right.$ VERA $\left.^{1}\right)$ project and the Bar and Spiral Structure Legacy $\left(\mathrm{BeSSeL}^{2}\right)$ survey have elucidated important aspects of the Milky Way galaxy, including values of its fundamental parameters and the nature of its spiral structure (Brunthaler et al. 2011; Reid et al. 2014; Honma et al. 2015; Sakai et al. 2015; $\mathrm{Xu}$ et al. 2016). The BeSSeL survey continues with additional VLBI observations of masers associated with High Mass StarForming Regions (HMSFRs) to better constrain the size and morphology of the Milky Way (see, e.g., Quiroga-Nuñez et al. 2017; Sanna et al. 2017). This is relevant at large Galactocentric radii $(>12 \mathrm{kpc})$, where only a few HMSFRs have been observed and their astrometric parameters are harder to measure (Hachisuka et al. 2015, and references within). Also, the outer Galactic region is particularly interesting as it gauges the Galactic rotation curve, which is a crucial key to understand the role of dark matter in Galactic dynamics (see, e.g., Kent 1986; Sofue 2017).

\footnotetext{
1 VERA is part of the National Astronomical Observatory of Japan.

2 http://bessel.vlbi-astrometry.org/
}

In 2004, the VERA project started to monitor several maser bearing stars and star-forming regions to accurately determine their astrometric parameters (Honma 2013). Their first result was the parallax and proper motion of the star-forming region Sharpless 269 (S 269), also known as Sh2-269, LBN 196.49-0.160 or G196.45-01.67 (Honma et al. 2007). S 269 is a compact HII region in the outer Galaxy toward the Galactic anticenter at $l=196.5$ and $b=-1.7$ (Sharpless 1959). It hosts several bright near-infrared (NIR) sources, in particular S 269 IRS 2w. This is a massive young O star with associated Herbig-Haro objects (Eiroa et al. 1994) and several species of masers (Minier et al. 2002; Sawada-Satoh et al. 2013). Water $(22 \mathrm{GHz})$, methanol $(6.7$ and $12.2 \mathrm{GHz})$ and $\mathrm{OH}(1.6 \mathrm{GHz})$ maser emission around S 269 IRS $2 \mathrm{w}$ have been detected and studied for decades (Clegg 1993; Minier et al. 2002; Lekht et al. 2001a) as the region presents signposts of star-forming activity (Jiang et al. 2003; Sawada-Satoh et al. 2013) and intermediate scale interstellar turbulence (Lekht et al. 2001b). S 269, therefore, represents one of a few well observed HII regions at large Galactocentric radii ( $>13 \mathrm{kpc}$, Honma et al. 2007).

Using the VERA array, Honma et al. (2007) monitored the water maser emission from S 269 IRS 2w from 2004 to 2006. 
They reported strong maser emission of $480 \mathrm{Jy}$ at $22 \mathrm{GHz}$ with $V_{\mathrm{LSR}}=19.7 \mathrm{~km} \mathrm{~s}^{-1}$, and measured an annual parallax of $0.189 \pm 0.008$ mas, corresponding to a distance from the Sun of $5.28_{-0.22}^{+0.24} \mathrm{kpc}$ and a Galactic rotational velocity similar to the Sun. This result suggested that the rotation curve of the Galaxy remains flat out to $13.5 \mathrm{kpc}$ from the Galactic center (adopting $R_{\odot}=8.34 \mathrm{kpc}$, Reid et al. 2014).

Later Miyoshi et al. (2012) and Asaki et al. (2014) disputed the distance to S 269 reported by Honma et al. (2007), firstly pointing out that kinematic and optical photometric distance estimates reported shorter values (3.7-3.8 kpc, see Moffat et al. 1979; Wouterloot \& Brand 1989; Xu et al. 2009). Moreover, they reanalyzed the VERA data specifically using more compact maser spots than those used by Honma et al. (2007), and reported a parallax value $0.247 \pm 0.034$ mas, which corresponds to a distance of $4.05_{-0.49}^{+0.65} \mathrm{kpc}$ (Asaki et al. 2014). The tension between the two parallax distances is crucial for two reasons. First, the S 269 astrometric parameters have been used to constrain the Galactic rotation curve at large Galactocentric radii due to the limited number of sources with accurately measured distances in this area of the Galaxy. Second, the two distance estimates support a different structure of the outer spiral arm. The nearer distance estimate of $4.05_{-0.49}^{+0.65} \mathrm{kpc}$ by Asaki et al. (2014) is inconsistent with previous distance estimates of the outer arm (Hachisuka et al. 2015), suggesting a kink or bifurcation, whereas the larger distance of $5.28_{-0.22}^{+0.24} \mathrm{kpc}$ by Honma et al. (2007) supports a smoother arm.

We now present the results and implications of a large number of recent Very Long Baseline Array (VLBA) observations of the $\mathrm{S} 269$ region at $22 \mathrm{GHz}$. In Sect. 2, we describe the observations, the data reduction procedure and the methods used. The astrometric results and the search for optical members within the Gaia catalog are described in Sect. 3. Then, in Sect. 4, we analyze the maser emission structure, and the implications of the parallax and proper motion obtained regarding the structure of the outer arm and optical associations. Finally, we present the main conclusions of this work in Sect. 5.

\section{Observations}

From August 2015 to October 2016, we conducted 16 epochs of phase reference observations of water masers present in S 269, using two extragalactic continuum sources (J0613+1306 and $\mathrm{J} 0619+1454)$ as close by position references at $0.73^{\circ}$ and $1.67^{\circ}$, respectively, from $S 269$. The observations were made using the VLBA operated by the National Radio Astronomy Observatory $\left(\mathrm{NRAO}^{3}\right)$ under program BR210E. Table A.1 shows the dates and times of the 16 observations, which correspond to a sequence of four observations (i.e., one in late summer, two in late winter or early spring, and one more in the next late summer) repeated four times, close in time during each sequence.

Four adjacent $16 \mathrm{MHz}$ bands, each in right and left circular polarization, were used with the third band centered on an $V_{\mathrm{LSR}}$ of $15 \mathrm{~km} \mathrm{~s}^{-1}$, assuming a rest frequency of the water maser $J_{K_{\mathrm{a}} K_{\mathrm{c}}}=6_{16} \rightarrow 5_{23}$ transition of $22235.080 \mathrm{MHz}$. The observations were processed with the VLBI software correlator VLBA$\mathrm{DiFX}^{4}$, producing 2000 and 32 spectral channels per band for

The National Radio Astronomy Observatory is a facility of the National Science Foundation operated under cooperative agreement by Associated Universities, Inc.

4 DiFX is developed as part of the Australian Major National Research Facilities Programme by the Swinburne University of Technology and operated under license.
Table 1. Information of the strongest S 269 maser spot detected, and both extragalactic sources used for parallax and proper motion estimate.

\begin{tabular}{cccc}
\hline \hline $\begin{array}{c}\text { Source } \\
\text { name }\end{array}$ & $\begin{array}{c}\alpha(\mathrm{J} 2000) \\
(\mathrm{hh}: \mathrm{mm}: \mathrm{ss})\end{array}$ & $\begin{array}{c}\delta(\mathrm{J} 2000) \\
\left({ }^{\circ}:^{\prime}:^{\prime}\right)\end{array}$ & $\begin{array}{c}S_{v}(\mathrm{Jy} \\
\left.\text { beam }^{-1}\right)\end{array}$ \\
\hline $\begin{array}{c}\text { S 269 } \\
\text { J0613+1306 }\end{array}$ & $06: 14: 37.6410$ & $+13: 49: 36.6930$ & 95.8 \\
$\mathrm{~J} 0619+1454$ & $06: 19: 52.8723$ & $+13: 06: 45.4010$ & 0.2 \\
\hline
\end{tabular}

Notes. S 269 spot is shown in Fig. 1 and corresponds to spot I in Fig. 2. The peak flux density corresponds to the observations made at Epoch $\mathrm{H}$.

the line and continuum data, respectively. This yielded a velocity spacing of $0.108 \mathrm{~km} \mathrm{~s}^{-1}$ for the line data. In addition, to estimate and then remove residual tropospheric delays relative to the correlator model, we inserted four geodetic blocks during each observational epoch (details about geodetic observations can be found in Reid et al. 2009). The observation cycles were designed such that S 269 was observed for 30 s (typically a 10 s slew and $20 \mathrm{~s}$ on source) followed by a compact extragalactic source for $30 \mathrm{~s}$. Therefore, the center of the maser scans used for phase referencing were $60 \mathrm{~s}$ apart, which is shorter than the coherence time for VLBA observations at this frequency. Positions and flux densities for the dominant maser $\operatorname{spot}\left(V_{\mathrm{LSR}}=19.6 \mathrm{~km} \mathrm{~s}^{-1}\right)$, used as the phase reference for the extragalactic sources, and the two continuum sources are shown in Table 1. The data reduction was performed using the NRAO Astronomical Image Processing System (AIPS), together with scripts written in ParselTongue (Kettenis et al. 2006), following standard BeSSeL survey data reduction methods (see Reid et al. 2009).

\section{Results}

Sixteen data cubes were constructed (one per epoch), each measuring 32768 pixels $\times 32768$ pixels $\times 300$ channels. This corresponds to an image of $1.64^{\prime \prime} \times 1.64^{\prime \prime}$ using a cellsize of 0.05 mas pixel $^{-1}$ within a radial velocity range between -6.4 and $25.7 \mathrm{~km} \mathrm{~s}^{-1}$. The range values for the data cube were calculated to include all the maser spots reported in Miyoshi et al. (2012) and Asaki et al. (2014).

We detected nine maser spots that were persistent for at least three epochs. Gaussian brightness distributions were fitted to the maser images by a least-squares method using the task JMFIT within AIPS. Table 2 shows the results of the fitting for each maser spot, together with its radial velocity and the maximum flux density across all epochs. Figure 1 shows the strongest water maser detected at representative epochs, while Fig. 2 shows the distribution of the maser spots, proper motion and radial velocities found in our VLBA data together with those reported in Honma et al. (2007) and Asaki et al. (2014). The strongest maser spot is labeled as "I" and it was used as central reference. In the VLBA observations, the water masers are confined to a radial velocity range between 16.0 and $20.4 \mathrm{~km} \mathrm{~s}^{-1}$. This is within the velocity range found in single dish spectra for S 269 (Lekht et al. 2001a).

\subsection{Elongated water maser emission}

The strongest maser spot was detected in all sixteen epochs with a flux density maximum of $95.8 \mathrm{Jy}_{\text {beam }}^{-1}$ at epoch $\mathrm{H}$. This spot has a distinctive elongated shape at all epochs (see Fig. 1), as was firstly reported by Miyoshi et al. (2012) and highlighted 
Table 2. Relative position, radial velocities and flux density peaks at certain observational epoch for the water maser spots shown in Fig. 2.

\begin{tabular}{rrrrrr}
\hline \hline $\begin{array}{r}\text { Spot ID } \\
\text { (Fig. 2) }\end{array}$ & $\begin{array}{r}\Delta \alpha \\
(\mathrm{mas})\end{array}$ & $\begin{array}{r}\Delta \delta \\
(\mathrm{mas})\end{array}$ & $\begin{array}{r}V_{\mathrm{LSR}} \\
\left(\mathrm{km} \mathrm{s}^{-1}\right)\end{array}$ & $\begin{array}{r}F_{\max } \\
\left(\mathrm{Jy} \mathrm{beam}^{-1}\right)\end{array}$ & $\begin{array}{r}\text { Peak } \\
\text { epoch }\end{array}$ \\
\hline I & $-0.101 \pm 0.005$ & $-0.020 \pm 0.002$ & 19.6 & 95.8 & $\mathrm{H}$ \\
II & $24.984 \pm 0.044$ & $4.116 \pm 0.024$ & 19.0 & 0.5 & $\mathrm{H}$ \\
III & $305.924 \pm 0.007$ & $4.652 \pm 0.012$ & 18.2 & 1.4 & $\mathrm{~B}$ \\
IV & $190.894 \pm 0.025$ & $-105.335 \pm 0.044$ & 20.4 & 0.3 & $\mathrm{D}$ \\
V & $109.886 \pm 0.006$ & $-143.253 \pm 0.010$ & 16.6 & 1.4 & $\mathrm{O}$ \\
VI & $-86.811 \pm 0.080$ & $-232.680 \pm 0.062$ & 19.8 & 12.9 & $\mathrm{H}$ \\
VII & $-586.729 \pm 0.011$ & $-666.299 \pm 0.005$ & 16.0 & 2.5 & $\mathrm{P}$ \\
VIII & $-758.007 \pm 0.009$ & $-741.869 \pm 0.013$ & 19.2 & 8.5 & $\mathrm{O}$ \\
IX & $-795.345 \pm 0.015$ & $-743.615 \pm 0.022$ & 17.4 & 0.6 & $\mathrm{~A}$ \\
\hline
\end{tabular}

Notes. The offsets were measured with respect to the strongest water maser spot, for which the absolute position is given in Table 1 . The epoch of fit was taken as the middle time of the VLBA observations, which is 2016.2.

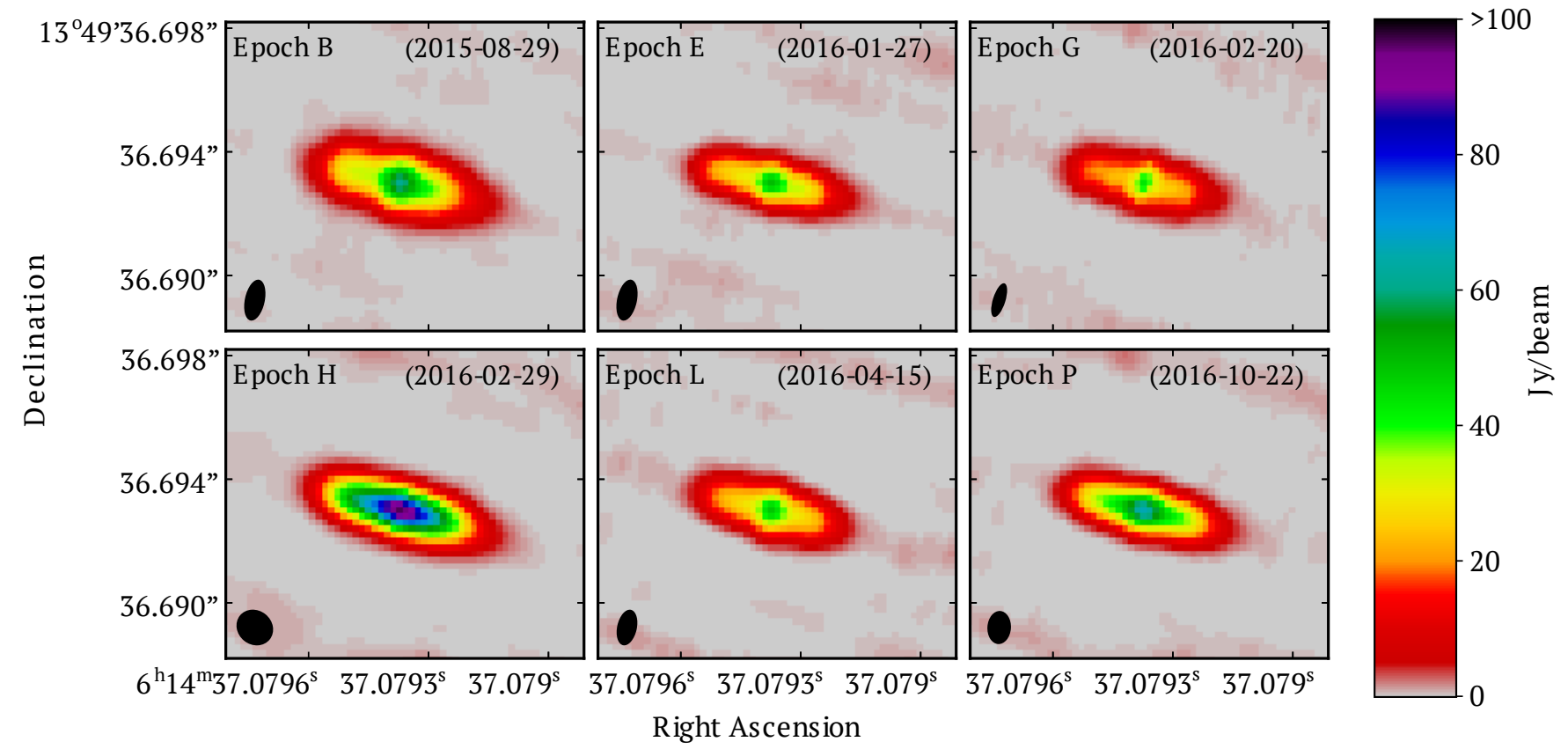

Fig. 1. Strongest $22 \mathrm{GHz}$ maser spot in S 269 region showing an elongated shape at six different epochs in the VLBA observations between 2015 and 2016. Based on its position and $V_{\mathrm{LSR}}$ (but not its motion, see Sect. 4.1), it seems to correspond to the strongest maser spot reported by Honma et al. (2007), using VERA observations in 2004 and 2005. This maser spot corresponds to the spot I in Fig. 2. The shape and size of the beam for each epoch is shown in the bottom left corner.

by Asaki et al. (2014). The spot varies somewhat over time but retains its basic shape throughout all our observations.

The inner core for this elongated spot could be well fitted by a single, compact, Gaussian brightness distribution, and we used the AIPS task JMFIT with a 2 mas box to fit the core.

\subsection{Astrometric measurements for $S 269$}

Only four of the nine $22 \mathrm{GHz}$ water maser spots were detected in at least ten epochs, which allow a robust fitting for the annual parallax sinusoidal signature in right ascension and declination. We also added error floor values to the position uncertainties in both sky coordinates and adjusted them to obtain $\chi_{v}^{2} \approx 1$ (see details in Reid et al. 2009).

The four maser spots used in the parallax fitting are labeled in Fig. 2 as spots I, III, V and VII and were detected in 16, 15, 10 and 16 epochs, respectively. As the four spots gave consistent parallax results (including the elongated spot, see Table 3 and Fig. 3), we also have calculated a combined fit by simultaneously fitting all data (i.e., four spots measured for both quasars). This yields a combined annual parallax value of $0.241 \pm 0.012$ mas. The uncertainty in the parallax includes an additional scaling factor of $\sqrt{N}$, where $N$ is the number of maser spots used for the fit. This accounts for the correlated systematic position variations among maser spots caused by atmospheric effects (Reid et al. 2009).

To estimate an average proper motion of the region, we fixed the annual parallax (previously calculated with only four maser spots) and fit the proper motions for the nine masers with respect to both (labeled as combined in Table 3 ) continuum extragalactic sources. Then, we averaged the proper motions of all nine maser spots detected by a standard mean (labeled as average proper motion in Table 3 ). Moreover, we include a $5 \mathrm{~km} \mathrm{~s}^{-1}$ uncertainty that accounts for the uncertainty of the motion of the masers with 


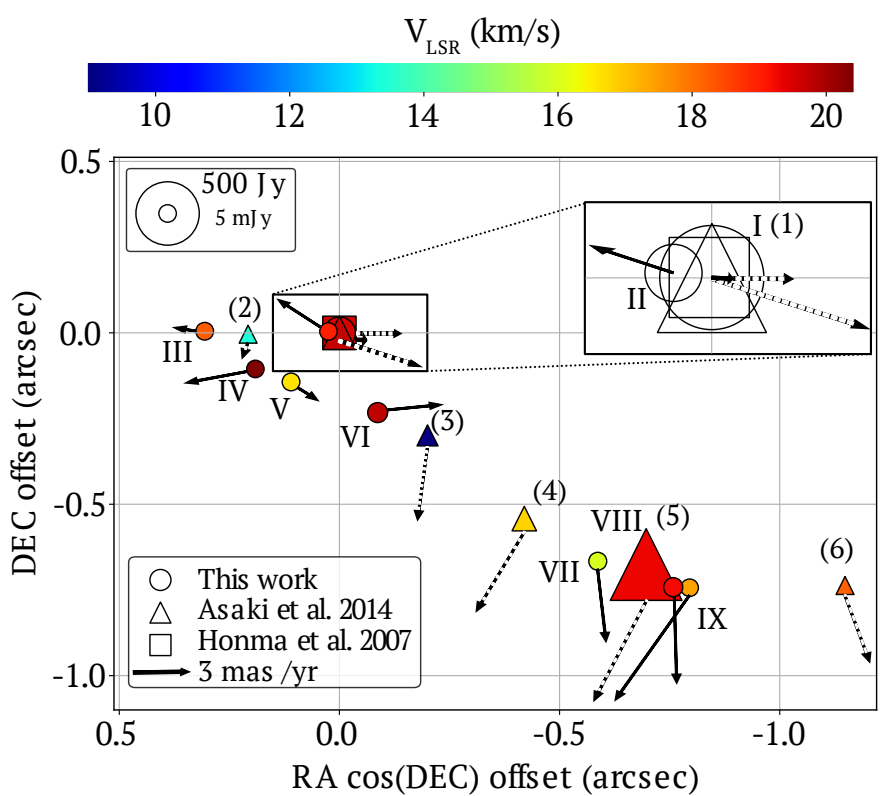

Fig. 2. Distribution of water maser spots around the strongest maser emission (where several maser spots coincide, see zoom in) with their radial velocity values expressed using a color scheme indicated by the bar at the top. The maser spots detected by the VLBA in 2015-2016 (see Table 2) are shown as circles, with Roman numbers and their proper motion as continuum arrows. Whereas, the main maser spots detected by VERA in 2004-2005 and published by Honma et al. (2007) and Asaki et al. (2014) are shown as squares and triangles, with Arabic numerals and their proper motion as dashed arrows. The size of the markers is proportional to the flux density peak of each maser spot (see upper left corner convention).

respect to the center of mass of the HMSFR. We note that the quiescent gas has $V_{\mathrm{LSR}}$ similar to the masers (i.e., 17.7, 16.5 and $18.2 \mathrm{~km} \mathrm{~s}^{-1}$ for CO, [SII] and HCN, respectively, Carpenter et al. 1990; Godbout et al. 1997; Pirogov 1999). Finally, we estimate $\mu_{\alpha} \cos \delta=0.16 \pm 0.26$ mas yr $^{-1}$ and $\mu_{\delta}=-0.51 \pm 0.26$ mas yr $^{-1}$ for the average proper motion of S 269 .

\subsection{Cross-matching with Gaia DR2}

S 269 IRS $2 w$ is located in a CO molecular cloud with a projected size of $7^{\prime} \times 10^{\prime}$ (Heydari-Malayeri et al. 1982; Carpenter et al. 1990). Other massive young stars, which could belong to the same stellar association, are expected to be detected in the vicinity of the CO molecular cloud. Since molecular gas is mostly confined to the Galactic plane (and mainly in the Galactic spiral arms) in a layer with FWHM of several hundred pc for Galactic radii greater than $10 \mathrm{kpc}$ (Heyer \& Dame 2015), we searched in the Gaia DR2 catalog within a spherical region around S 269's location in 3D. As GMCs usually extend from 5 pc up to 120 pc, with a very few exceptional cases over $150 \mathrm{pc}$ (Murray 2011), we used a radius $125 \mathrm{pc}$ (1.73 at S 269's distance) as a conservative value to guarantee that most of the plausible sources associated with the S 269 region were included in the inspected range. This corresponds to a parallax range (including $\pm \sigma$ ) from 0.2225 to 0.2615 mas. Figure 4 shows the S 269 region using data from the Digital Sky Survey 2 (DSS2 ${ }^{5}$, Lasker \& McLean 1994) and the Two Micron All Sky Survey (2MASS ${ }^{6}$, Skrutskie et al. 2006) centered on the maser emission.

\footnotetext{
5 http://archive.eso.org/dss/dss

6 https://www.ipac.caltech.edu/2mass/
}

We only selected sources with confident parallax measurements in Gaia DR2 (i.e. $\sigma_{\pi} / \pi<0.2$ ) that allow direct distance estimates (Bailer-Jones 2015). In total, there are 2279 sources that fall into the spherical region defined. The closest ten Gaia counterparts in 3D are highlighted in red in Fig. 4, and their astrometric information is shown in Table A.2. We did not find an optical counterpart in Gaia DR2 that corresponds to the massive young star which surrounding material is yielding the water maser emission detected at $22 \mathrm{GHz}$. This is expected for a newly forming star that is deeply embedded in its placental material. However, the three closest optical counter parts (first three rows in Table A.2) were found within a core size of the $\mathrm{S} 269 \mathrm{HII}$ region $(3.9 \mathrm{pc} \times 2.8 \mathrm{pc})$ estimated by Godbout et al. (1997). These three Gaia DR2 sources have an average parallax that differs with respect to the VLBA observations by $-32 \pm 23 \mu$ as (assuming a Gaia zero-point correction of $\sim-0.03$ mas) and an average proper motion that differs by $0.02 \pm 0.65$ and $-0.16 \pm 0.77 \operatorname{mas~yr}^{-1}$ for $\mu_{\alpha} \cos \delta$ and $\mu_{\delta}$ respectively. Therefore, they are likely members of the stellar association that contains S 269 IRS 2w.

\section{Discussion}

\subsection{Long-lived and extended water maser emission}

\subsubsection{Elongated maser spot}

The unusual morphology of the spot I over many (from VERA in 2004 to VLBA in 2016) observations (Fig. 1) compared to typical maser spots vouches for its authenticity. An instrumental artifact, instead, would manifest a similar structural appearance in all similarly calibrated maser emission in the data cube, which is not the present case. We further analyzed the maser structure using DIFmap's modelfit and projplot tools. The structure of the maser is well fit by an elongated structure $\left(\mathrm{PA} \sim 78^{\circ}\right)$ plus a compact core as it is evident in Fig. 1.

\subsubsection{Correspondence with previous water maser observations}

As a collisionally pumped maser transition, $22 \mathrm{GHz}$ water masers are typically found in turbulent regions of postshocked gas associated with star formation outflows (see, e.g., Liljestrom \& Gwinn 2000; Hollenbach et al. 2013, and the references within). While a shocked region at some radial velocity may consistently produce maser emission around the shock velocity, the individual maser spots are typically shortlived ( $\lesssim 1 \mathrm{yr}$, see e.g., Tarter \& Welch 1986). With this in mind, the persistent appearance of the maser spot I in Fig. 2 at about the same location in the source makes it remarkable. Its position and shape seem to correspond to the maser spot reported by Honma et al. (2007) for the VERA observations made between 2004 and 2005, and the reanalysis of VERA data made by Miyoshi et al. (2012) and Asaki et al. (2014). Its longevity may be related to its complex structure, maser spots typically being much more compact. In principle, this could allow us to fit the parallax and proper motion over a 10 year baseline for this spot.

Although this region seems to persistently yield elongated maser emission over decades, indicating it is the same masering cloud, there is a significant difference between the proper motion measured for the observing set in 2004 using VERA and 2015 using the VLBA, with respect to the same quasar (J0613+1306). On the one hand, Honma et al. (2007) reported for the elongated 
Table 3. Fitting results of parallax and proper motion for four $22 \mathrm{GHz}$ water maser spots present in $\mathrm{S} 269$ with respect to two extragalactic continuum sources, under the usual assumption that the quasars are stationary during our observations.

\begin{tabular}{|c|c|c|c|c|c|c|c|}
\hline $\begin{array}{c}\text { Maser } \\
\text { spot }\end{array}$ & Quasar & $\begin{array}{c}\pi \\
\text { (mas) }\end{array}$ & $\begin{array}{r}\mu_{\alpha} \cos \delta \\
\left(\operatorname{mas~yr}^{-1}\right) \\
\end{array}$ & $\begin{array}{r}\mu_{\delta} \\
\left(\mathrm{mas} \mathrm{yr}^{-1}\right) \\
\end{array}$ & $\begin{array}{c}V_{\mathrm{LSR}} \\
\left(\mathrm{km} \mathrm{s}^{-1}\right) \\
\end{array}$ & $\begin{array}{r}\text { Combined } \\
\mu_{\alpha} \cos \delta\left(\mathrm{mas} \mathrm{yr}^{-1}\right) \\
\end{array}$ & $\begin{array}{r}\text { Combined } \\
\mu_{\delta}\left(\mathrm{mas} \mathrm{yr}^{-1}\right) \\
\end{array}$ \\
\hline \multirow[t]{2}{*}{ I } & J0613+1306 & $0.244 \pm 0.010$ & $-0.091 \pm 0.024$ & $-0.003 \pm 0.029$ & 19.6 & $-0.099 \pm 0.019$ & $-0.008 \pm 0.020$ \\
\hline & $\mathrm{J} 0619+1454$ & $0.243 \pm 0.013$ & $-0.107 \pm 0.032$ & $-0.012 \pm 0.028$ & & & \\
\hline \multirow[t]{2}{*}{ II } & J0613+1306 & - & $0.518 \pm 0.187$ & $0.408 \pm 0.099$ & 19.0 & $0.507 \pm 0.128$ & $0.438 \pm 0.076$ \\
\hline & J0619+1454 & & $0.497 \pm 0.187$ & $0.464 \pm 0.124$ & & & \\
\hline \multirow[t]{2}{*}{ III } & J0613+1306 & $0.226 \pm 0.013$ & $0.173 \pm 0.033$ & $0.049 \pm 0.039$ & 18.2 & $0.170 \pm 0.025$ & $0.063 \pm 0.028$ \\
\hline & J0619+1454 & $0.228 \pm 0.017$ & $0.166 \pm 0.041$ & $0.078 \pm 0.042$ & & & \\
\hline \multirow[t]{2}{*}{ IV } & J0613+1306 & - & $0.767 \pm 0.115$ & $-0.234 \pm 0.194$ & 20.4 & $0.763 \pm 0.089$ & $-0.185 \pm 0.139$ \\
\hline & J0619+1454 & & $0.762 \pm 0.144$ & $-0.137 \pm 0.206$ & & & \\
\hline \multirow[t]{2}{*}{ V } & J0613+1306 & $0.234 \pm 0.033$ & $-0.189 \pm 0.032$ & $-0.177 \pm 0.057$ & 16.6 & $-0.187 \pm 0.052$ & $-0.177 \pm 0.057$ \\
\hline & J0619+1454 & $0.214 \pm 0.042$ & $-0.108 \pm 0.133$ & $-0.207 \pm 0.085$ & & & \\
\hline \multirow[t]{2}{*}{ VI } & J0613+1306 & - & $-0.639 \pm 0.215$ & $0.085 \pm 0.068$ & 19.8 & $-0.650 \pm 0.147$ & $0.080 \pm 0.050$ \\
\hline & J0619+1454 & & $-0.661 \pm 0.212$ & $0.069 \pm 0.082$ & & & \\
\hline \multirow[t]{2}{*}{ VII } & J0613+1306 & $0.254 \pm 0.026$ & $-0.108 \pm 0.064$ & $-1.255 \pm 0.034$ & 16.0 & $-0.111 \pm 0.043$ & $-1.254 \pm 0.024$ \\
\hline & J0619+1454 & $0.248 \pm 0.024$ & $-0.115 \pm 0.060$ & $-1.254 \pm 0.035$ & & & \\
\hline \multirow[t]{2}{*}{ VIII } & J0613+1306 & - & $-0.209 \pm 0.286$ & $-1.602 \pm 0.490$ & 19.2 & $-0.031 \pm 0.160$ & $-1.580 \pm 0.250$ \\
\hline & J0619+1454 & & $0.152 \pm 0.120$ & $-1.542 \pm 0.347$ & & & \\
\hline \multirow[t]{2}{*}{ IX } & J0613+1306 & - & $0.950 \pm 0.819$ & $-2.142 \pm 0.213$ & 17.4 & $1.052 \pm 0.529$ & $-1.959 \pm 0.139$ \\
\hline & J0619+1454 & & $1.149 \pm 0.839$ & $-1.772 \pm 0.140$ & & & \\
\hline $\begin{array}{l}\text { Combined } \\
\text { parallax }\end{array}$ & & $0.241 \pm 0.012$ & & $\begin{array}{r}\text { Average } \\
\text { proper motion }\end{array}$ & & $0.157 \pm 0.066$ & $-0.509 \pm 0.037$ \\
\hline
\end{tabular}

Notes. Radial velocities of those masers are also shown. The description of how the combined parallax and proper motion were obtained is described in Sect. 3.2. The first column gives the maser spot number used in Fig. 2. The annual parallax values $(\pi)$ provided in the third column were fitted for only four masers (see Sect. 3.2). For the absolute proper motion fittings (Cols. 4,5,7,8), we fixed the parallax value in $0.241 \pm 0.012$ mas. The procedures to obtain the combined and average values reported in this table are described in Sect. 3.2.

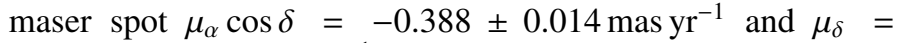
$-0.118 \pm 0.071 \mathrm{mas} \mathrm{yr}^{-1}$, and Asaki et al. (2014) reported $-0.738 \pm 0.008$ mas yr$^{-1}$ and $-0.249 \pm 0.007$ mas yr$^{-1}$ (Spot ID 6 in Asaki et al. 2014). In contrast, we estimated a much slower

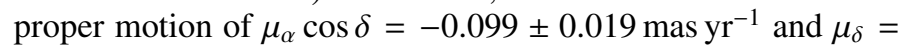
$-0.008 \pm 0.020$ mas $^{-1}$. It seems likely that small changes in the coherent amplification path of the maser account for these differences. Thus, we cannot fit the parallax and proper motion for this spot on a 10 year baseline given its velocity discrepancy, but its peculiar morphology hints that those spots represent the same maser region.

\subsubsection{Source of the elongated water maser emission}

In principle, an amplified background source could mimic the particular properties (morphology and longevity) of the elongated maser spot, however, there is no sign of such continuum source when we inspected the continuum bands of our observations. Alternatively, the linear distribution of the water maser spots (Fig. 2) suggests that we are observing the front shock of the outflow moving in the southeastern direction. This direction is confirmed by infrared data from the Simultaneous-3color InfraRed Imager for Unbiased Survey (SIRIUS), where S 269 IRS 2 shows a bipolar jet in the southeastern-northwestern direction (Jiang et al. 2003, but it remains unclear if it is associated with S269 IRS $2 \mathrm{w}$ or S 269 IRS 2e). This fact suggests that material may have been compressed yielding an elongated maser emission, which indeed is perpendicular to the shock motion. Moreover, CO, [SII] and HCN observations of the $\mathrm{S} 269$ region reported a $V_{\mathrm{LSR}}$ of $17.7,16.5$ and $18.2 \mathrm{~km} \mathrm{~s}^{-1}$, respectively (Carpenter et al. 1990; Godbout et al. 1997; Pirogov 1999), which differs with respect to our maser observations supporting the jet origin of the maser emission.

\subsubsection{Cyclic maser emission in S 269}

Lekht et al. (2001a) monitored the water maser emission toward S 269 for more than 20 years (1980-2001) using the 22 meter telescope of the Pushchino Radio Astronomy Observatory. They reported a $V_{\mathrm{LSR}}$ range of $[19.6-20.4] \mathrm{km} \mathrm{s}^{-1}$ in which our VLBA observations and also those made by Honma et al. (2007) fall. Although this single dish effort could not image the water maser, they found that the integrated flux of the strongest maser had cyclic emission between 70 and $600 \mathrm{Jy}$ with a period of between 4.8 and 6.6 years. Assuming the cyclic emission suggested by Lekht et al. (2001a), subsequent peak emissions (over $200 \mathrm{Jy}$ ) should have occurred between [2004.4-2006.2], [2009.2-2012.8] and [2014-2019.4]. Both VERA (Honma et al. 2007) and VLBA (this work) observations spanned more than one year within these time ranges, but only VERA observations showed enhanced emission of $480 \mathrm{Jy}$. Although there is evidence of previous flares at radio wavelengths in S 269 (see, e.g., Clegg 1993), the cyclic emission proposed by Lekht et al. (2001a) does not seem consistent with our VLBA observations.

\section{2. $S 269$ astrometric parameters}

\subsubsection{Distance}

The combined fit of the four $22 \mathrm{GHz}$ water maser spots presented in Table 3 yielded a parallax value of $0.241 \pm 0.012$ mas, which corresponds to a distance of $4.15_{-0.20}^{+0.22} \mathrm{kpc}$ from the Sun 

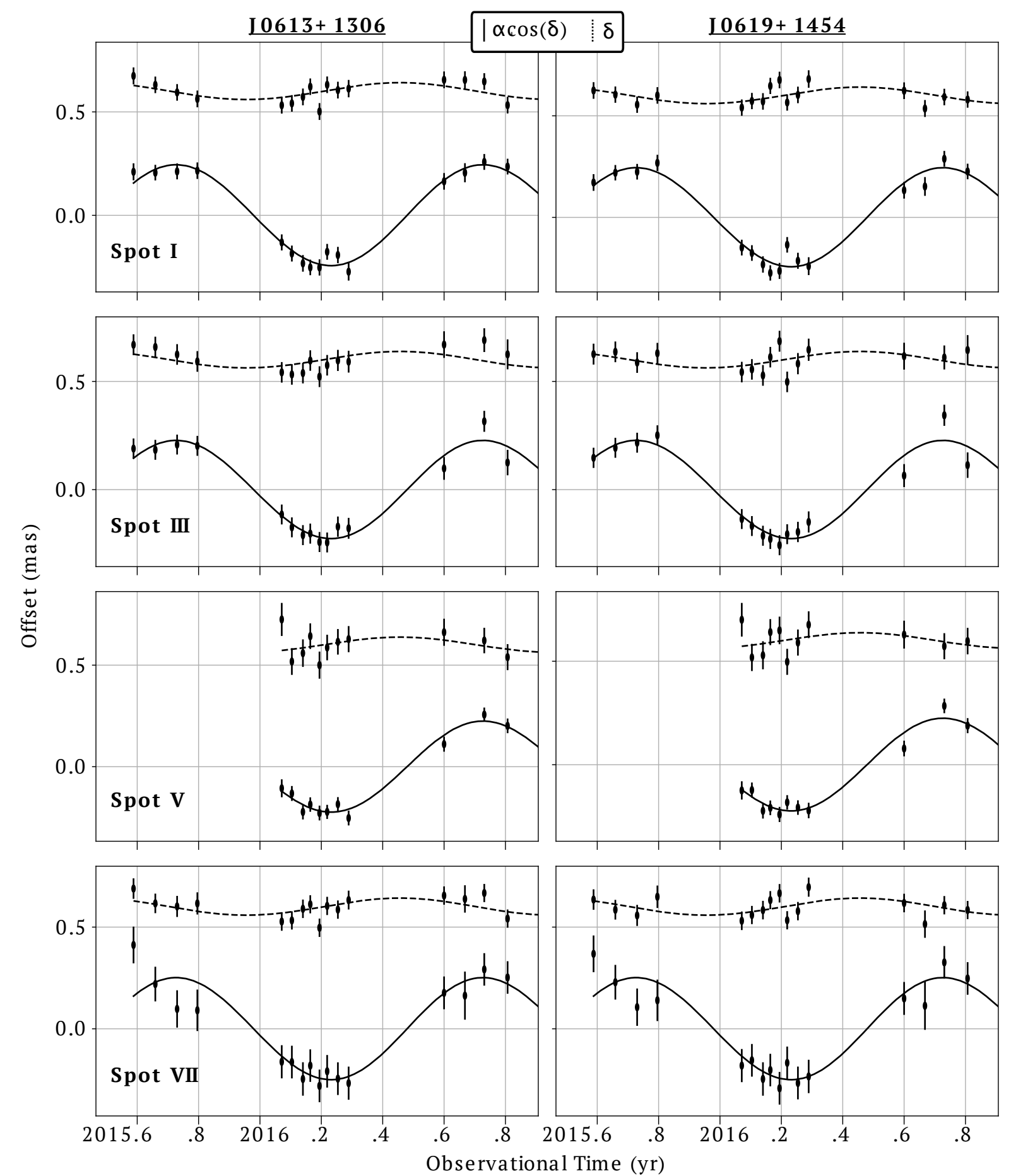

Fig. 3. Astrometric offsets for four different water masers with respect to the quasars used as reference position: J0613+1306 in the left plots and J0619+1454 in the right plots. The proper motions were subtracted from the parallax signatures. The solid and dashed lines represent the eastward $(\alpha \cos \delta)$ and northward $(\delta)$ individual fitting listed in Table 3, respectively. The Northward offset $(\delta)$ was shifted +0.6 mas for visualization purposes.

and $12.36 \pm 0.27 \mathrm{kpc}$ from the Galactic center (adopting $R_{\odot}=$ $8.34 \mathrm{kpc}$, Reid et al. 2014). The annual parallax is in agreement with $0.247 \pm 0.034$ mas obtained by Asaki et al. (2014) for the VERA data taken between 2004 and 2005.

Although Honma et al. (2007) reported a smaller annual parallax of $0.189 \pm 0.008$ mas, and hence a larger distance of $5.28_{-0.22}^{+0.24} \mathrm{kpc}$ for the elongated maser spot, Asaki et al. (2014) claimed that VERA data for that single spot yielded an inexact parallax estimate. They suggested that this problematic morphology caused an erratic positional estimate. Indeed, VERA baselines are short and few compared to the VLBA, and therefore they could not resolve and fit the inner core of the elongated spot. However, with the new VLBA observations, we have been able to fit and constrain the annual parallax to the compact core $(0.244 \pm 0.012$ mas $)$ with respect to both extragalactic sources (see Table 3). This fact could explain the distance discrepancy between Honma et al. (2007) and this work's measurement. Also, as it was mentioned by Asaki et al. (2014), parallax 

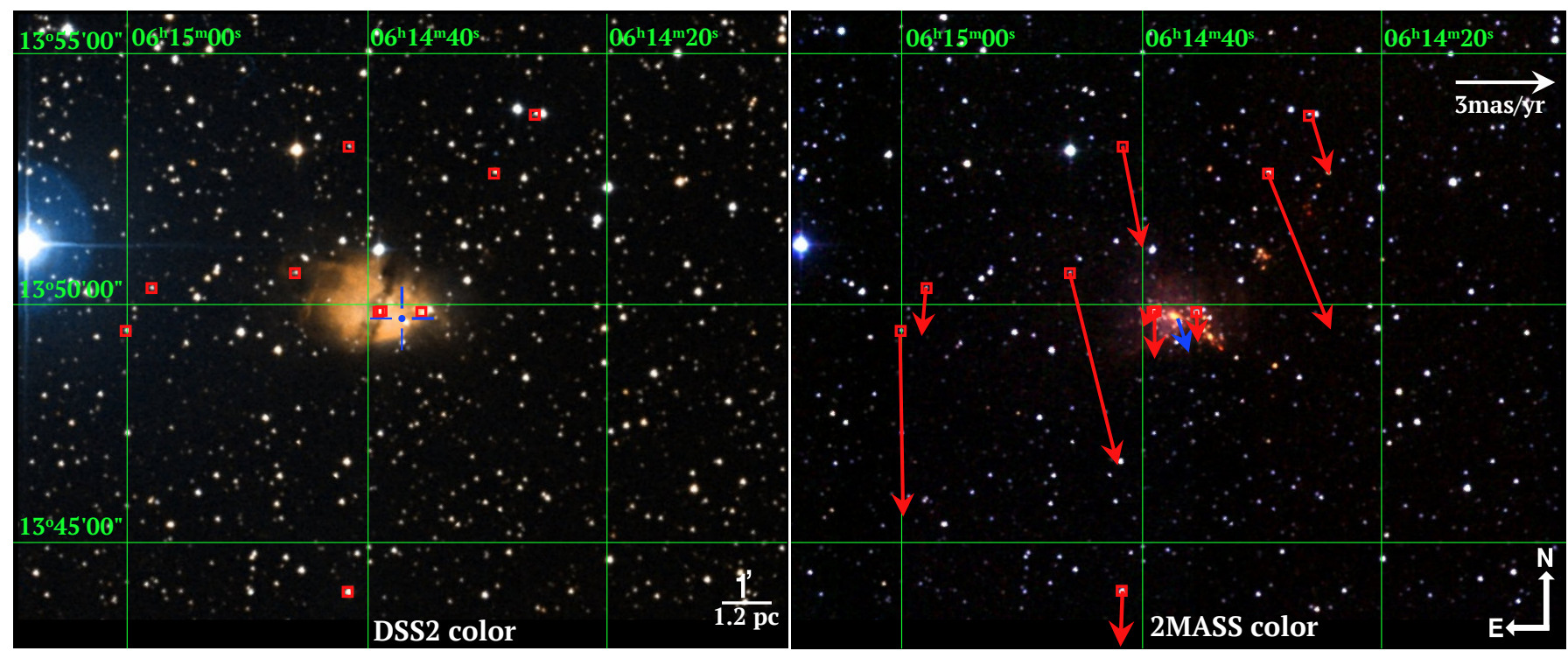

Fig. 4. Image of the S 269 region using data from DSS2 (left panel) and 2MASS (right panel) with a sky projected size of $15.65^{\prime} \times 12.33^{\prime}$, centered on the water maser emission (blue central sign) detected with the VLBA (Table 1), that seems to be triggered by the massive young star S 269 IRS 2w (Asaki et al. 2014). Left panel: the molecular cloud appears as a butterfly with two wings separated by a dark fringe where a B0.5 star has been detected in the center (Moffat et al. 1979). In contrast, near-infrared observations (right panel) show that the center of the clustered region of massive young objects coincides with the position of S 269 IRS $2 \mathrm{w}$. The red squares are the closest optical sources around S 269 found within the Gaia DR2 catalog (see Sects. 3.3 and 4.5) and their astrometric information is shown in Table A.2. The red arrows display the proper motions for such sources, whereas the blue arrow is the proper motion calculated by us using the water masers in the region. The images were generated with the Aladdin interface (Bonnarel et al. 2000), where the color map descriptions of DSS2 and 2MASS can be found through alasky.u-strasbg.fr/DSS/DSSColor/ and alasky.u-strasbg.fr/2MASS/Color/.

uncertainties reported by Honma et al. (2007) might be larger than quoted as the possibility of correlated positional variations among the three spots used was not considered.

\subsubsection{Peculiar velocity}

We transformed the estimated 3D average motion of the maser

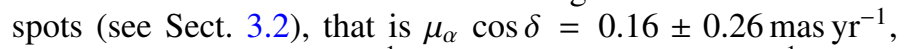
$\mu_{\delta}=-0.51 \pm 0.26 \mathrm{mas} \mathrm{yr}^{-1}$ and $V_{\mathrm{LSR}}=19.6 \pm 5 \mathrm{~km} \mathrm{~s}^{-1}$, to the $(U, V, W)$ reference frame that rotates with the Galactic disk, yielding $U_{\mathrm{S} 269}=3 \pm 5, V_{\mathrm{S} 269}=-1 \pm 5$ and $W_{\mathrm{S} 269}=6 \pm 5$ in $\mathrm{km} \mathrm{s}^{-1}$, where $U$ increases toward the Galactic center, $V$ in the direction of Galactic rotation and $W$ toward the north Galactic pole. We assumed a rotation model defined by Reid et al. (2014) with $R_{0}=8.31 \mathrm{kpc}$ and $\Theta_{0}=241 \mathrm{~km} \mathrm{~s}^{-1}$, $U_{\odot}=10.5 \mathrm{~km} \mathrm{~s}^{-1}, \quad V_{\odot}=14.4 \mathrm{~km} \mathrm{~s}^{-1}, \quad W_{\odot}=8.9 \mathrm{~km} \mathrm{~s}^{-1}$ and $\mathrm{d} \Theta / \mathrm{d} R=-0.2 \mathrm{~km} \mathrm{~s}^{-1} \mathrm{kpc}^{-1}$. The obtained values are consistent with previous findings of near-zero peculiar motion for water masers associated with HMSFRs (Reid et al. 2014).

The tangential motion of S 269 allows us to constrain the Galactic rotation at $12.4 \mathrm{kpc}$ radius from the center of the Milky Way. The errors in $V_{\mathrm{S} 269}$ reported in this work are comparable to those reported by Honma et al. (2007), where a different model for the Galactic rotation was used. As a consequence, we find that the S 269 tangential motion is within $2 \%$ of a flat Galactic rotation curve, as it was initially claimed by Honma et al. (2007), albeit at a larger distance compared with this work.

\subsection{Membership in the Perseus or outer arm}

In order to investigate whether S 269 lies within a spiral arm, we generated simulations of Galactic maser sources following the model proposed by Quiroga-Nuñez et al. (2017). Although that model was initially developed for methanol masers associ- ated with HMSFRs, it can be used to estimate the kinematics of other masers at certain regions of the Galaxy. There are three differences with respect to the model that Quiroga-Nuñez et al. (2017) implemented. First, we did not consider any luminosity function for water masers, since it is not necessary for our kinematic study. Second, we populated the phase space of our model with many more (up to half million) sources to allow an accurate sampling. Third, the spiral structure model follows the arm description derived by Reid et al. (2014). Although this spiral structure model was obtained using the S 269's distance estimated by Honma et al. (2007), S 269 was not the only source used for the spiral structure model, and also, this model was a smooth extension of the spiral arm from the first to the second quadrant. Figure 5 shows the simulated distribution that was obtained by this way but displaying only 2000 sources for plotting purposes.

The phase-space density distributions of masers simulated for the outer Galaxy is shown in Fig. 6, with the observational values of S 269 plotted. For the distributions, all the variables are measured from the Sun and simulated errors in these observables were also generated (see Quiroga-Nuñez et al. 2017, for details). In all cases, the kinematic parameters of S 269 seem to suggest that this source is more likely member of the outer arm than the Perseus arm, as was pointed out by Sakai et al. (2012) based on the distance estimate made by Honma et al. (2007).

\subsection{Outer arm structure}

Previous pitch angle estimates for the outer arm (e.g., Reid et al. 2014) were obtained based on the large distance to S 269 published by Honma et al. (2007). Moreover, several sources were excluded from the pitch angle fit since they were considered interarm sources (Reid et al. 2014; Hachisuka et al. 2015). To investigate this, we recalculated the outer arm position using 


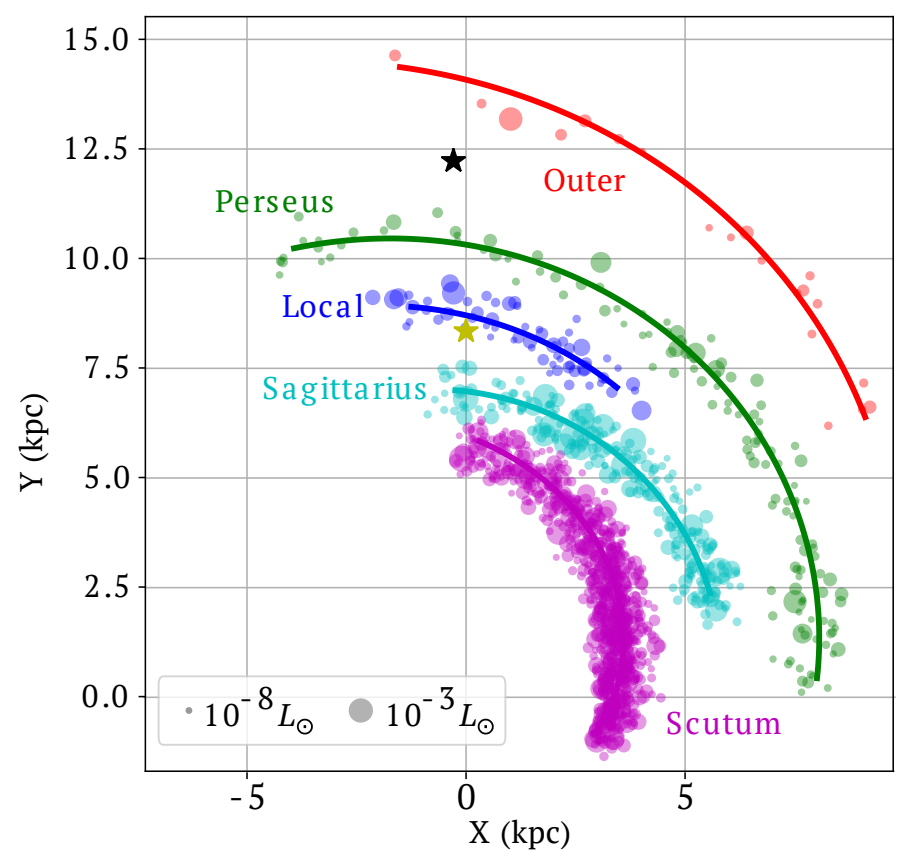

Fig. 5. Plan view of a simulation of the Galactic maser distribution for maser bearing stars around the spiral structure using the model developed by Quiroga-Nuñez et al. (2017). The spiral structure estimated by Reid et al. (2014) was populated with artificial sources to compare the phase-space density distribution of the spiral arms with S 269 properties (see Sect. 4.3). The Galactic center is located at $(0,0)$, and the yellow and black stars correspond to the position of the Sun (Reid et al. 2014) and S 269, respectively, where their positional error bars are smaller than the size of the marker.

recent astrometric information from ten other HMSFRs coming from the BeSSeL survey (Reid priv. comm.). These sources seem to belong to the outer arm based on their kinematics and parallaxes (see Table 4). We assess three possible scenarios for the outer arm: a single arm with a constant pitch angle, an arm with two segments that form a kink where they join, and an arm that bifurcates. In all cases, the new fit locates the outer arm in the third quadrant closer to the Sun, compared to what was previously reported (Sanna et al. 2012; Reid et al. 2014).

\subsubsection{A single arm}

Following the procedure in Reid et al. (2009) and assuming a width of $0.63 \pm 0.18 \mathrm{kpc}$ for the outer arm (estimated by Reid et al. 2014), we fitted 11 sources (see Table 4), finding that the spiral arm can be described using the form:

$\ln (R)=(2.50 \pm 0.02)-(\pi / 180)(\beta-17.9) \tan (\Psi)$,

where $R$ is the Galactocentric radii in kpc at a Galactocentric azimuth $\beta$ (which is zero toward the Sun and increases with Galactic longitude) and $\Psi$ the pitch angle with a value of $6.2 \pm 3^{\circ}$. 1 . This description applies for $73^{\circ} \lesssim l \lesssim 218^{\circ}$, which corresponds to the Galactic longitude range of the sources used.

Figure 7 shows a plan view of the Milky Way, where the spiral arm positions estimated by Reid et al. (2014) are shown as black curves for reference. The pitch angle for the outer arm calculated by Reid et al. (2014) (i.e. $13.8 \pm 3^{\circ} .3$ ) is within the errors compared to other published values based on masers associated with massive young objects (e.g., Sanna et al. (2012) and Hachisuka et al. (2015) reported $12.1 \pm 4.2$ and $14.9 \pm$ 2.7 , respectively). In contrast, the outer arm position with our estimate of the pitch angle (i.e., $6.2 \pm 3^{\circ} .1$ ) is shown in the same figure as a green line. This pitch angle is unusually small compared with previous studies -even without considering S 269 as an outer arm source- and entirely attributed to the sources at large Galactic longitudes $\left(>140^{\circ}\right)$ suggesting that a kink in the outer arm is another plausible explanation. Finally, although the outer arm sampling used is sparse, the reconstruction of the arm is still the best procedure with the limited astrometric solutions available.

\subsubsection{Two arm segments forming a kink}

Honig \& Reid (2015) analyzed the positions of a large number of HII regions in four face-on galaxies, and concluded that spiral arms seem to be composed of segments that join up and sometimes produce abrupt changes in pitch angle (kinks). We have tested if the outer arm presents a similar feature by splitting the sample into two balanced subsamples, that is five sources with $l<140^{\circ}$ and six sources with $l>140^{\circ}$. We estimated a pitch angle for the first segment $\left(l<140^{\circ}\right) 10^{\circ} .5 \pm 5^{\circ} .9$, and $7^{\circ} .9 \pm 5.8$ for the second segment $\left(l>140^{\circ}\right)$. The fits to both segments are shown in Fig. 7. While, with the small number of sources, the pitch angle estimates are quite uncertain, Fig. 7 suggests either a kink or bifurcation in the outer arm somewhere near a longitude of $l \sim 140^{\circ}$. Note also that this representation calls for a kink with a change of pitch angle of $\gtrsim 25 \%(\Delta \Psi /|\Psi|)$, comparable to values of $\sim 20 \%$ which are common in spiral galaxies (Savchenko \& Reshetnikov 2013). Moreover, the position of the outer arm observed in HI maps by Koo et al. (2017) for the third quadrant requires a significant displacement (or kink) within the range of $140^{\circ}<l<210^{\circ}$. Clearly, more sources with accurate measurements are needed to refine the position of a possible kink in the outer arm.

\subsubsection{Bifurcation of the arm}

As mentioned above, looking at the parallax positions of sources in Fig. 7, one could hypothesize that some sources follow the outer arm model of Reid et al. (2014) into quadrant 3, while others rather follow the new single arm model with a smaller pitch angle or the segmented arm model, forming thus a bifurcation at $l \sim 140^{\circ}$. Although HI maps of the Milky Way suggest that bifurcations of the Galactic arms (e.g., Koo et al. 2017) might occur, we cannot establish if this is the case for the outer arm at the Galactic longitudes investigated here, especially in the Galactic anticenter direction, where HI maps are inaccurate due to the largest velocity component (caused by the Galactic rotation) not being radial but transversal with respect to the Sun. More sources are needed to evaluate the likelihood of this hypothesis.

\subsection{Optical members of the same stellar association}

Massive young stars are understood to be formed from Giant Molecular Clouds that collapse generating high- and low-mass stellar cores (e.g., Tan et al. 2014). We can search for associated stars using Gaia DR2, but given that the HMSFR that hosts the S 269 IRS 2w massive young star is located close to the Galactic plane $(b=-1.46)$, and at $4.15 \mathrm{kpc}$ from the Sun, only the brightest, early-type members of the same stellar association are expected to be detectable with Gaia.

We review the proper motion for the stars within $125 \mathrm{pc}$ around S 269 (see Sect. 3.3) using the Gaia DR2, finding that the closest ( $\sim 37$ pc projected distance) stellar cluster is NGC 2194. The Gaia parallax for NGC 2194 (i.e., $0.232 \pm 0.027$ calculated 


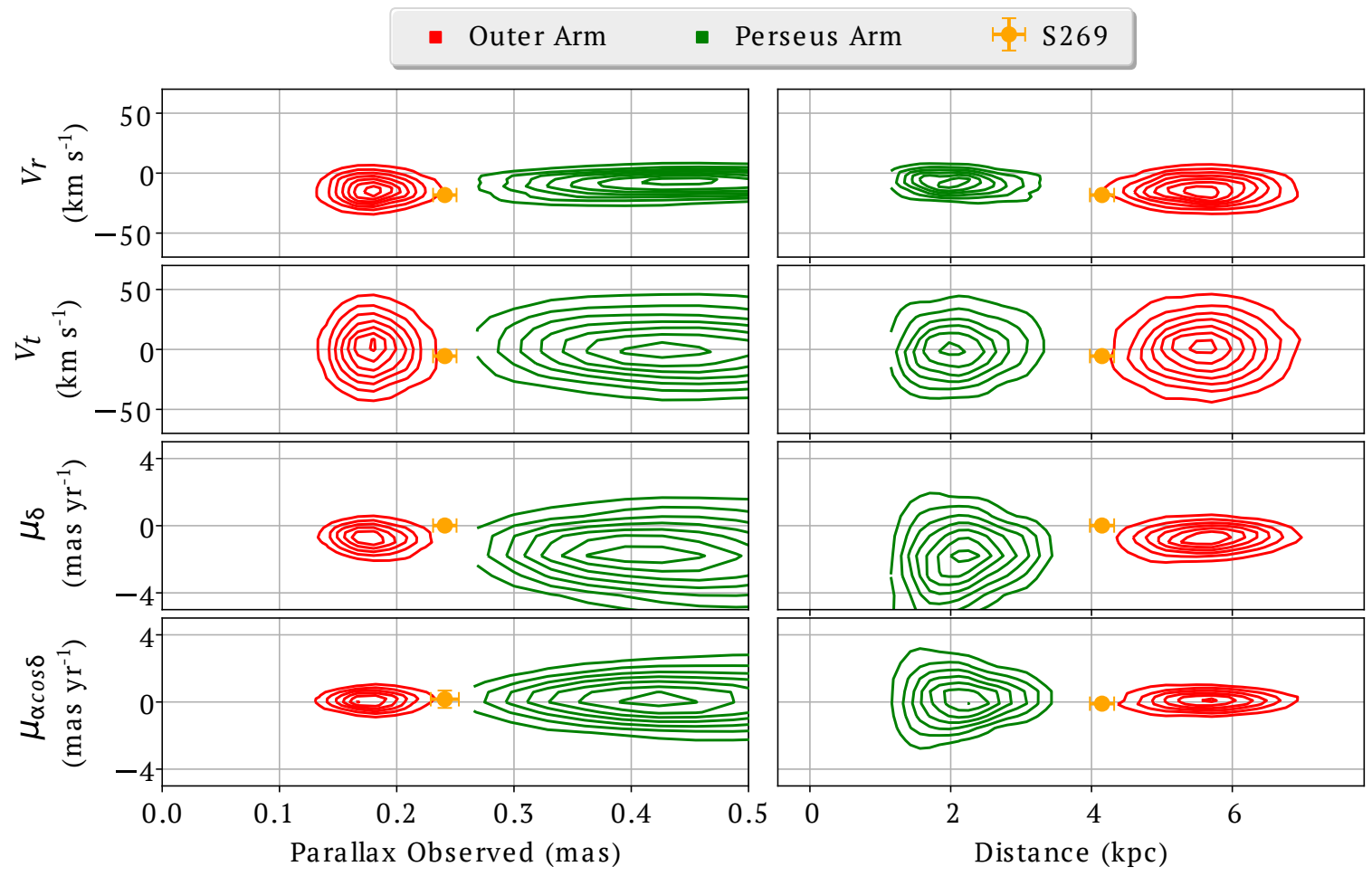

Fig. 6. Radial velocity $\left(V_{\mathrm{r}}\right)$, transversal velocity $\left(V_{\mathrm{t}}\right)$ and proper motion for $\mathrm{S} 269$ seen from the Sun as a function of observed parallax (left plots) and distance (right plots). The contours show the distribution of sources per arm for a simulated Galactic maser distribution around the position of S 269 where the pitch angles of each arm were constant (see Sect. 4.3). The simulations were made using the code developed by Quiroga-Nuñez et al. (2017). Each single contour represents a percentage of the arm sources contained in a specific region of the plot from the inside as $[0.14,0.28,0.42,0.56,0.70,0.84,0.98]$.

Table 4. Astrometric information for 11 HMSFRs obtained with the VLBA in the outer arm region.

\begin{tabular}{lcccc}
\hline \hline Name & $\begin{array}{c}\alpha \\
\text { (hh:mm:ss) }\end{array}$ & $\begin{array}{c}\delta \\
\left({ }^{\circ}:^{\prime \prime}\right)\end{array}$ & $\begin{array}{c}\pi \\
\text { (mas) }\end{array}$ & Ref. \\
\hline G073.65+00.19 & $20: 16: 21.932$ & $+35: 36: 06.094$ & $0.075 \pm 0.020$ & $*$ \\
G075.30+01.32 & $20: 16: 16.012$ & $+37: 35: 45.810$ & $0.108 \pm 0.010$ & 1 \\
G090.92+01.48 & $21: 09: 12.969$ & $+50: 01: 03.664$ & $0.171 \pm 0.031$ & $*$ \\
G097.53+03.18 & $21: 32: 12.434$ & $+55: 53: 49.689$ & $0.133 \pm 0.017$ & 2 \\
G135.27+02.79 & $02: 43: 28.568$ & $+62: 57: 08.388$ & $0.167 \pm 0.011$ & 7 \\
G160.14+03.16 & $05: 01: 40.244$ & $+47: 07: 19.026$ & $0.244 \pm 0.006$ & $*$ \\
G168.06+00.82 & $05: 17: 13.744$ & $+39: 22: 19.915$ & $0.187 \pm 0.022$ & 2,3 \\
G182.67-03.26 & $05: 39: 28.425$ & $+24: 56: 31.946$ & $0.157 \pm 0.042$ & 2,4 \\
G196.45-01.68 (S 269) & $06: 14: 37.641$ & $+13: 49: 36.693$ & $0.242 \pm 0.011$ & 5 \\
G211.60+01.06 & $06: 52: 45.321$ & $+01: 40: 23.072$ & $0.239 \pm 0.010$ & $*$ \\
V838 Mon & $07: 04: 04.822$ & $-03: 50: 50.636$ & $0.163 \pm 0.016$ & 6 \\
\hline
\end{tabular}

Notes. These sources were used for the outer arm fitting (Sect. 4.3). The names include the galactic coordinates except for V838 Mon which is $(217.80,+01.05)$. The parallax of each source might differ from the published values in the references as we combined independent measurements (one per reference) to increase their accuracy. The parallaxes marked with * will be published as part of the BeSSeL survey (Reid priv. comm.).

References. (1) Sanna et al. (2012), (2) Hachisuka et al. (2015), (3) Honma et al. (2011), (4) data reanalyzed of Hachisuka et al. (2015), (5) variance averaged between Asaki et al. (2014) and results of Table 3 (6) Sparks et al. (2008), (7) Hachisuka et al. (2009).

for 217 stellar members with $\sigma_{\pi} / \pi<20 \%$ including zero-point correction of -0.03 mas) is consistent with the $\mathrm{S} 269$ parallax. However, there are several reasons to suggest that S 269 may not be directly associated with NGC 2194. First, based on chemical composition, Amado et al. (2004) and Netopil et al. (2016) have estimated an age of $0.87 \pm 0.19 \mathrm{Gyr}$ and $0.60 \pm 0.25 \mathrm{Gyr}$ for NGC 2194, whereas HMSFRs are expect to be two orders of magnitude younger (see, e.g., Battersby et al. 2017). Indeed, Jiang et al. (2003) reported a dynamic age of $10^{5} \mathrm{yr}$ for S 269 .
Second, there seems to be a serious discrepancy between the published luminosity distance $(1.9 \pm 0.1 \mathrm{kpc}$, Jacobson et al. 2011) and the distance estimate that one can obtain with Gaia data.

Finally, the three closest Gaia sources to S 269 IRS 2w found within the core of the S 269 HII region defined by Godbout et al. (1997) (i.e., $3.9 \mathrm{pc} \times 2.8 \mathrm{pc}$ ) correspond to the three first rows in Table A.2. Given that these sources have an average parallax and proper motion that are consistent with respect to the 


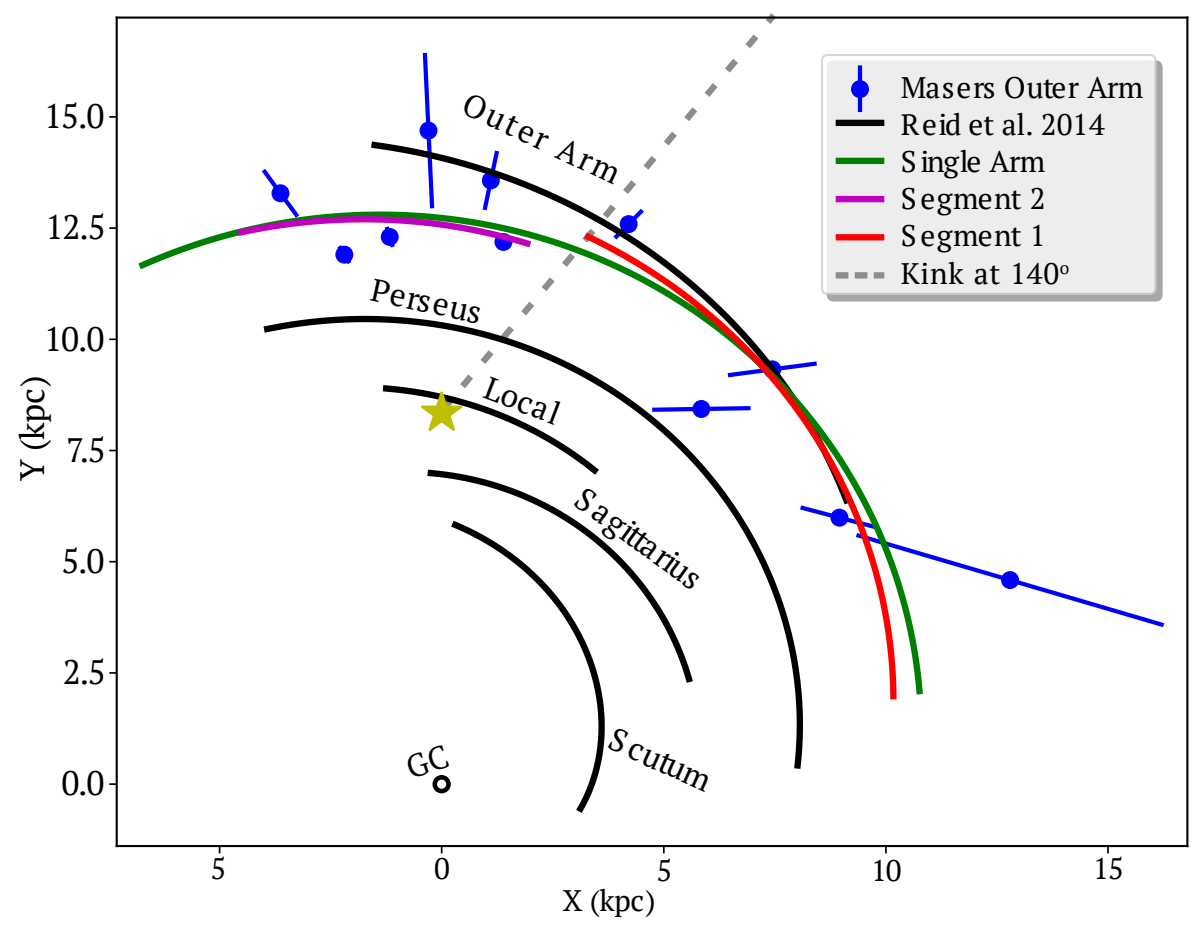

Fig. 7. Plan view of Galactic spiral structure. The spiral structure estimated by Reid et al. (2014) is shown as black lines for reference. Maser emission from 11 HMSFRs has been used to estimate the position of the outer arm. The different outer arm descriptions discussed in Sect. 4.3 are highlighted in color curves. The Galactic center is located at $(0,0)$, and the yellow star corresponds to the Solar position (Reid et al. 2014), while the orange line demarcates the latitude of the outer arm kink suggested (i.e., $140^{\circ}$ ).
VLBA observations (i.e., $-32 \pm 23 \mu$ as, $0.02 \pm 0.65$ and $-0.16 \pm$ $0.77 \mathrm{mas} \mathrm{yr}^{-1}$ ), we suggest that they are likely early-type members of the same stellar association that contains S 269 IRS $2 \mathrm{w}$. However, further studies of these companion stars and their reddening could be used to estimate the age of S 269 and possibly refine its astrometry.

\section{Conclusions}

We present the results of high-accuracy VLBA observations of the $\mathrm{S} 269$ region using relative astrometry. We detected nine water maser spots in S 269 that were prominent during at least three observing epochs. Four maser spots were detected in at least ten epochs, which allows a precise annual parallax fitting of $0.241 \pm 0.012$ mas corresponding to a distance of $4.15_{-0.20}^{+0.22} \mathrm{kpc}$.

Although the calculated distance corroborates the results previously published by Asaki et al. (2014), we show that the strongest maser spot (which was left out from their analysis because of its elongated shape) yields a well-constrained annual parallax, when the inner core position is used for the fit. Also, the longevity of the elongated water maser spot in the region is remarkable as it spans more than ten years (i.e., 2004-2016, between Honma et al. 2007, and our observations), however given the significant changes in proper motion between both observational sets, we could not estimate a 10-year astrometric fit. In addition, the VLBA images and the distribution of maser spots indicate that this spot could be originated from the compression of material in a shock front that propagates perpendicular to the elongation. Moreover, water maser emission detected in the same region from 1980 to 2001 by Lekht et al. (2001b) is likely to be the same that the VLBA detected in 2015-2016. However, the cyclic emission period previously estimated does not seem consistent with our observations.

We calculated a Galactic peculiar velocity for $\mathrm{S} 269$ to be $(2 \pm 6,4 \pm 14,4 \pm 13) \mathrm{km} \mathrm{s}^{-1}$ in the $(U, V, W)$ Galactic frame, which confirms that the rotation curve at large radii $(\sim 12.4 \mathrm{kpc})$ is fairly flat. On the other hand, since there is no model that ties the masers in a shock front to the motion of the underlying star, the accuracy with which we know the motion of the system is limited.

By comparing S269's position and proper motion with respect to other sources in the outer region of the Milky Way, we fitted the outer arm position, locating it closer to the Sun than previously thought. We explored three different scenarios: a new single outer arm pitch angle of $6.2 \pm 3$. 1 , a kink in the outer arm between two different segments and a bifurcation of the arm. Although all three are plausible explanations, the low value of a single arm pitch angle with respect to other arms and the lack of astrometric information to test a secondary segment coming from a bifurcation, lead us to favor a kink model. This kink can be described by two segments with pitch angles of $7.9 \pm 5.8$ and $10.5 \pm 5^{\circ} .9$, locating the kink in the second quadrant $\left(\sim 140^{\circ}\right)$. This explanation is consistent with $\mathrm{HI}$ maps at $l>180^{\circ}$, and is also supported by observations of similar features in other galaxies. Future observations are needed to assess if this is the case for the outer arm.

Finally, the Gaia DR2 catalog was inspected around S 269 for optical companions, which could be members of the stellar association. We did not find an optical counterpart for S 269 IRS 2w which could be exciting the water maser emission. However, we did find three optical sources that are likely members of the same stellar association that contains S 269 IRS 2w. Moreover, only one cluster (NGC 2194) was detected in the vicinity, but it is unlikely to be associated with S 269 given the difference in age. Future explorations of optical associations with respect to VLBI astrometric data are planned (e.g., Pihlström et al. 2018) to refine the criteria for optical stellar companions around HMSFRs and evolved stars.

Acknowledgements. The National Radio Astronomy Observatory is a facility of the National Science Foundation operated under cooperative agreement by Associated Universities, Inc. This work made use of the Swinburne University of Technology software correlator, developed as part of the Australian Major National Research Facilities Programme and operated under license. This work has also made use of data from the European Space Agency (ESA) mission Gaia (https://www.cosmos.esa.int/gaia), processed by the Gaia Data Processing and Analysis Consortium (DPAC, https://www.cosmos.esa.int/ 
web/gaia/dpac/consortium). Funding for the DPAC has been provided by national institutions, in particular the institutions participating in the Gaia Multilateral Agreement. Moreover, this research has made use of "Aladin sky atlas" developed at CDS (http://cds.u-strasbg.fr/), Strasbourg Observatory, France. The Digitized Sky Survey was produced at the Space Telescope Science Institute under US Government grant NAG W-2166. The images of these surveys are based on photographic data obtained using the Oschin Schmidt Telescope on Palomar Mountain and the UK Schmidt Telescope. The plates were processed into the present compressed digital form with the permission of these institutions. This publication makes use of data products from the Two Micron All Sky Survey, which is a joint project of the University of Massachusetts and the Infrared Processing and Analysis Center/California Institute of Technology, funded by the National Aeronautics and Space Administration and the Nationa Science Foundation. The authors sincerely acknowledge the anonymous referee for the suggestions that have improved this manuscript. L.H.Q.-N. would also deeply thank Dr. A.G.A Brown at Leiden Observatory for his comments and suggestions regarding the Gaia cross-match.

\section{References}

Amado, P. J., Suárez, J. C., Moya, A., et al. 2004, A\&A, 414, 163 Asaki, Y., Imai, H., Sobolev, A. M., \& Yu Parfenov, S. 2014, ApJ, 787, 54 Bailer-Jones, C. A. L. 2015, PASP, 127, 994

Battersby, C., Bally, J., \& Svoboda, B. 2017, ApJ, 835, 263

Bonnarel, F., Fernique, P., Bienaymé, O., et al. 2000, A\&AS, 143, 33

Brunthaler, A., Reid, M., Menten, K., et al. 2011, Astron. Nachr., 332, 461

Carpenter, J. M., Snell, R. L., \& Schloerb, F. P. 1990, ApJ, 362, 147

Clegg, A. W. 1993, in Astrophysical Masers, eds. A. W. Clegg, \& G. E. Nedoluha (Berlin, Heidelberg: Springer, Berlin Heidelberg), 279

Eiroa, C., Casali, M., Miranda, L., \& Ortiz, E. 1994, A\&A, 290, 599

Godbout, S., Joncas, G., Durand, D., \& Arsenault, R. 1997, ApJ, 478, 271

Hachisuka, K., Brunthaler, A., Menten, K. M., et al. 2009, ApJ, 696, 1981

Hachisuka, K., Choi, Y. K., Reid, M. J., et al. 2015, ApJ, 800, 2

Heydari-Malayeri, M., Testor, G., Baudry, A., Lafon, G., \& de La Noe, J. 1982, A\&A, 113, 118

Heyer, M., \& Dame, T. 2015, ARA\&A, 53, 583

Hollenbach, D., Elitzur, M., \& McKee, C. F. 2013, ApJ, 773, 70

Honig, Z. N., \& Reid, M. J. 2015, ApJ, 800, 53

Honma, M. 2013, New Trends Radio Astron ALMA Era 30th Anniv Nobeyama Radio Obs Proc a Symp held Hakone, 476, 81

Honma, M., Bushimata, T., Choi, Y. K., et al. 2007, PASJ, 59, 889
Honma, M., Hirota, T., Kan-Ya, Y., et al. 2011, PASJ, 63, 17

Honma, M., Nagayama, T., \& Sakai, N. 2015, PASJ, 67, 70

Jacobson, H. R., Pilachowski, C. A., \& Friel, E. D. 2011, AJ, 142, 59

Jiang, Z., Yao, Y., \& Yang, J. 2003, ApJ, 596, 1064

Kent, S. M. 1986, AJ, 91, 1301

Kettenis, M., van Langevelde, H. J., Reynolds, C., \& Cotton, B. 2006, in Astron. Data Anal. Softw. Syst. XV, ASP Conf. Ser., 351, 497

Koo, B. C., Park, G., Kim, W. T., et al. 2017, PASP, 129, 094102

Lasker, B. M., \& McLean, B. J. 1994, STSci Newsl., 11, 39

Lekht, E. E., Pashchenko, M. I., \& Berulis, I. I. 2001a, Astron. Reports Transl. from Astron. 1 Zhurnal Orig. Russ. Text Copyr. c, 45, 949

Lekht, E. E., Silant'ev, N. A., Mendoza-Torres, J. E., Pashchenko, M. I., \& Krasnov, V. V. 2001b, A\&A, 377, 999

Liljestrom, T., \& Gwinn, C. R. 2000, ApJ, 10, 781

Minier, V., Booth, R. S., \& Conway, J. E. 2002, A\&A, 383, 614

Miyoshi, M., Asaki, Y., Wada, K., \& Imai, H. 2012, New Astron., 17, 553

Moffat, A. F. J., Fitzgerald, M. P., \& Jackson, P. D. 1979, A\&AS, 38, 197

Murray, N. 2011, ApJ, 729, 133

Netopil, M., Paunzen, E., Heiter, U., \& Soubiran, C. 2016, A\&A, 585, A150

Pihlström, Y. M., Sjouwerman, L. O., Claussen, M. J., et al. 2018, ApJ, 868, 72

Pirogov, L. 1999, A\&A, 348, 600

Quiroga-Nuñez, L. H., Van Langevelde, H. J., Reid, M. J., \& Green, J. A. 2017, A\&A, 604, A72

Reid, M. J., Menten, K. M., Brunthaler, A., et al. 2009, ApJ, 693, 397

Reid, M., Menten, K., Brunthaler, A., et al. 2014, ApJ, 783, 130

Sakai, N., Honma, M., Nakanishi, H., et al. 2012, PASJ, 64, 108

Sakai, N., Nakanishi, H., Matsuo, M., et al. 2015, PASJ, 67, 69

Sanna, A., Reid, M. J., Dame, T. M., et al. 2012, ApJ, 745, 82

Sanna, A., Reid, M. J., Dame, T. M., Menten, K. M., \& Brunthaler, A. 2017, Science, 358, 227

Savchenko, S. S., \& Reshetnikov, V. P. 2013, MNRAS, 436, 1074

Sawada-Satoh, S., Fujisawa, K., Sugiyama, K., Wajima, K., \& Honma, M. 2013, PASJ, 65, 79

Sharpless, S. 1959, ApJS, 4, 257

Skrutskie, M. F., Cutri, R. M., Stiening, R., et al. 2006, ApJ, 131, 1163

Sofue, Y. 2017, PASJ, 69, 1

Sparks, W. B., Bond, H. E., Cracraft, M., et al. 2008, AJ, 135, 605

Tan, J. C., Beltran, M. T., Caselli, P., et al. 2014, Protostars Planets VI, 149

Tarter, J. C., \& Welch, W. J. 1986, ApJ, 305, 467

Wouterloot, J., \& Brand, J. 1989, A\&AS, 80, 149

Xu, Y., Voronkov, M. A., Pandian, J. D., et al. 2009, A\&A, 507, 1117

Xu, Y., Reid, M., Dame, T., et al. 2016, Sci. Adv., 2, e1600878 


\section{Appendix A: Additional tables}

Table A.1. VLBA observational epochs for S 269 as part of the BR210E program during 2015-2016.

\begin{tabular}{|c|c|c|}
\hline Epoch & $\begin{array}{c}\text { Date } \\
(\mathrm{dd} / \mathrm{mm} / \text { yyyy })\end{array}$ & $\begin{array}{c}\text { Time range (UTC) } \\
\text { (hh:mm:ss-hh:mm:ss) }\end{array}$ \\
\hline A & 03/Aug/2015 & $11: 55: 29-18: 57: 42$ \\
\hline B & 29/Aug/2015 & 10:13:15-17:15:28 \\
\hline $\mathrm{C}$ & 24/Sep/2015 & 08:31:02-15:33:14 \\
\hline $\mathrm{D}$ & $18 /$ Oct/2015 & $06: 56: 40-13: 58: 53$ \\
\hline $\mathrm{E}$ & 27/Jan/2016 & $00: 19: 33-07: 19: 45$ \\
\hline $\mathrm{F}$ & 07/Feb/2016 & $23: 32: 22-06: 32: 34$ \\
\hline G & 20/Feb/2016 & $22: 41: 15-05: 41: 27$ \\
\hline $\mathrm{H}$ & 29/Feb/2016 & 22:05:52-05:06:04 \\
\hline I & 11/Mar/2016 & $21: 22: 37-04: 22: 49$ \\
\hline $\mathrm{J}$ & 20/Mar/2016 & $20: 47: 14-03: 47: 26$ \\
\hline $\mathrm{K}$ & 02/Apr/2016 & $19: 56: 07-02: 56: 19$ \\
\hline $\mathrm{L}$ & 15/Apr/2016 & $19: 05: 00-02: 05: 12$ \\
\hline M & 07/Aug/2016 & $11: 36: 47-18: 36: 58$ \\
\hline $\mathrm{N}$ & 01/Sep/2016 & 09:58:29-16:58:40 \\
\hline $\mathrm{O}$ & 24/Sep/2016 & $08: 28: 03-15: 28: 14$ \\
\hline $\mathrm{P}$ & $22 / \mathrm{Oct} / 2016$ & 06:37:58-13:38:09 \\
\hline
\end{tabular}

Table A.2. Astrometric parameters, mean $g$ apparent magnitude and color given by Gaia DR2 for ten optical sources found in the vicinity of S 269 $\left(10^{\prime} \times 7^{\prime}\right.$, Carpenter et al. 1990) or $12.1 \mathrm{pc} \times 8.4 \mathrm{pc}$ assuming our distance estimate of $4.15 \mathrm{kpc}$.

\begin{tabular}{cccccrc}
\hline \hline $\begin{array}{c}\text { Gaia } \\
\text { source ID }\end{array}$ & $\begin{array}{c}\alpha(\mathrm{J} 2015.5) \\
(\mathrm{hh}: \mathrm{mm}: \mathrm{ss})\end{array}$ & $\begin{array}{c}\delta(\mathrm{J} 2015.5) \\
\left({ }^{\circ}:^{\prime \prime}:^{\prime}\right)\end{array}$ & $\begin{array}{c}d_{\mathrm{S} 269} \\
(\operatorname{arcmin})\end{array}$ & $\begin{array}{c}\text { Parallax } \\
(\mathrm{mas})\end{array}$ & $\begin{array}{r}\mu_{\alpha} \cos \delta \\
\left(\mathrm{mas} \mathrm{yr}^{-1}\right)\end{array}$ & $\begin{array}{c}\mu_{\delta} \\
\left(\mathrm{mas} \mathrm{yr}^{-1}\right)\end{array}$ \\
\hline 3344575631369939200 & $06: 14: 38.73$ & $+13: 49: 45.06$ & 0.30 & $0.232 \pm 0.043$ & $-0.012 \pm 0.078$ & $-0.739 \pm 0.069$ \\
3344575627071194240 & $06: 14: 38.99$ & $+13: 49: 43.90$ & 0.35 & $0.247 \pm 0.028$ & $-0.113 \pm 0.052$ & $-0.247 \pm 0.046$ \\
3344578586307442560 & $06: 14: 35.35$ & $+13: 49: 43.40$ & 0.57 & $0.238 \pm 0.042$ & $0.020 \pm 0.077$ & $-0.491 \pm 0.068$ \\
3344575695792364672 & $06: 14: 45.94$ & $+13: 50: 30.56$ & 2.20 & $0.304 \pm 0.056$ & $0.711 \pm 0.097$ & $-2.908 \pm 0.088$ \\
3344578959966755200 & $06: 14: 29.28$ & $+13: 52: 32.46$ & 3.56 & $0.255 \pm 0.029$ & $0.919 \pm 0.058$ & $-2.379 \pm 0.052$ \\
3344580506155003776 & $06: 14: 41.50$ & $+13: 53: 05.03$ & 3.60 & $0.284 \pm 0.055$ & $0.280 \pm 0.099$ & $-1.547 \pm 0.089$ \\
3344576937039978624 & $06: 14: 57.98$ & $+13: 50: 12.24$ & 4.97 & $0.238 \pm 0.047$ & $-0.070 \pm 0.080$ & $-0.732 \pm 0.073$ \\
3344579784600525952 & $06: 14: 25.88$ & $+13: 53: 43.46$ & 5.01 & $0.260 \pm 0.037$ & $0.257 \pm 0.069$ & $-0.864 \pm 0.066$ \\
3344576181125735424 & $06: 15: 00.10$ & $+13: 49: 20.22$ & 5.46 & $0.262 \pm 0.034$ & $0.029 \pm 0.064$ & $-2.753 \pm 0.058$ \\
3344573909084277632 & $06: 14: 41.57$ & $+13: 44: 02.90$ & 5.64 & $0.279 \pm 0.025$ & $-0.031 \pm 0.043$ & $-0.854 \pm 0.038$ \\
\hline
\end{tabular}

Notes. The sky distribution of the sources is shown in optical and NIR images in Fig. 4. The projected distance $\left(d_{\mathrm{S} 269}\right)$ between the Gaia sources and maser position S 269 (06:14:37.6410,+13:49:36.6930). The sources are organized by proximity to the water mater emission with the closest first. The three first rows are the optical stars that were found within the size of the HII region S 269 defined by Godbout et al. (1997) as $3.9 \mathrm{pc} \times 2.8 \mathrm{pc}$ and are likely to be associated with the S 269 HII region (Sect. 4.5). 